\title{
Perception of the risk of sexual transmission of HIV among Congolese and Japanese university students
}

\author{
Nlandu Roger Ngatu • Ryoji Hirota $\cdot$ Masamitsu Eitoku • Basilua Andre Muzembo • Makoto Nishimori \\ Manatsu Kuramochi - Shuhei Shintani - Shin Inoue · Ruriko Takiuchi · Marina Maegawa • Daniel Ribble • \\ Muaka Anselme Mbenza • Nani-Tuma Hippolyte Situakibanza • Pita Damien Mbanzulu • Narufumi Suganuma
}

Received: 23 December 2010/Accepted: 24 July 2011/Published online: 23 August 2011

(C) The Japanese Society for Hygiene 2011

\begin{abstract}
Objective Sub-Saharan Africa remains the region most heavily affected by HIV infection. This study aimed to evaluate the knowledge, attitudes, and practices of Congolese students of risk behaviors for sexual transmission of HIV in comparison with their Japanese counterparts.

Methods Of the 1,747 undergraduate students who participated in the survey, there were 1,326 respondents (752 Japanese, 574 Congolese) who voluntarily and fully filled out the auto-administered questionnaire.

Results The proportion of Congolese respondents who do not always use condoms with an occasional sex partner was significantly higher, $57 \%$, as compared with their
\end{abstract}

N. R. Ngatu $(\bowtie) \cdot$ R. Hirota $\cdot$ M. Eitoku

B. A. Muzembo · N. Suganuma

Division of Social Medicine,

Department of Environmental Medicine,

Kochi Medical School, Kochi University,

Nankoku, Kochi 783-8505, Japan

e-mail: drngatu_roger@yahoo.fr

M. Nishimori - M. Kuramochi - S. Shintani · S. Inoue

R. Takiuchi - M. Maegawa $\cdot$ D. Ribble

Kochi Medical School, Kochi University, Kochi, Japan

M. A. Mbenza

University of Lubumbashi, Katanga,

Democratic Republic of Congo

N.-T. H. Situakibanza

Department of Medicine and Center for Tropical Diseases,

Faculty of Medicine, University of Kinshasa,

Kinshasa, Democratic Republic of Congo

P. D. Mbanzulu

Department of Gynecology and Obstetrics,

Faculty of Medicine, University of Kinshasa,

Kinshasa, Democratic Republic of Congo
Japanese counterparts $(15 \% ; p<0.001)$. Fewer than $40 \%$ $(9.9-39.7 \%)$ of Congolese respondents had accurate knowledge about multiple sex partnership (MSP), men sex with men (MSM), precocious sex, and commercial sex work (CSW) being high-risk behaviors $(p<0.001)$ for the sexual transmission of HIV infection. However, the proportion of Congolese tested or willing to get tested for HIV was significantly higher $(97.2 \%)$ than that in the Japanese group $(72.4 \%, p<0.001)$. In Congolese students, we observed an absence of adherence to preventive measures such as condom use with an occasional sex partner, and a greater proportion of students having inaccurate knowledge of major risk behaviors such as MSM, precocious sex, and MSP, compared with their Japanese counterparts.

Conclusions This study showed that, though sexual contact remains the main mode of HIV transmission in the region, Congolese students tend to have inaccurate knowledge of risk behaviors that expose people to the sexual transmission of HIV. This suggests that continuous education targeting those risk behaviors is of great importance to reduce the spread of the HIV epidemic.

Keywords HIV testing - Multiple sex partnership - Risk behavior - Sexually transmitted infection · Polygamy

\section{Introduction}

Human immunodeficiency virus (HIV) remains a global health problem of unprecedented dimensions and has already caused an estimated 25 million deaths worldwide. According to the World Health Organization (WHO), SubSaharan Africa remains the region most heavily affected by HIV infection, accounting for $67 \%$ of all people living with HIV and for 75\% of AIDS deaths in 2007 [1]. However, 
HIV infection prevention has been largely unsuccessful in many African countries [2, 3].

Knowing and targeting local risk factors/behaviors that contribute to the spread of the HIV epidemic in Sub-Saharan Africa would be a better way to help reduce the rate of new cases. Apart from illiteracy, which accounts for approximately $40 \%$ of the population or more, and poverty, locally specific risk behaviors and cultural background may possibly play a role in the progress of the disease in the region. Thus, we hypothesized that there might be socio-cultural factors that might contribute to the high HIV prevalence in SubSaharan African countries. Studies conducted in some developing countries have shown that younger age and illiteracy or a low education level were correlated with the risk of HIV infection and with poor knowledge of risk factors and the transmission of the disease [4-6].

In order to minimize the effect of illiteracy on the knowledge and attitudes of young people towards HIV, and given the fact that Japan has one of the lowest HIV prevalences in the world (less than $0.1 \%$ ), we conducted a survey among Congolese university students, with a higher education level than common people, and their Japanese counterparts. In this study, we sought to determine whether socio-cultural factors expose Congolese students to sexually transmitted infections (STIs) and HIV to a greater degree in comparison with their Japanese counterparts. We hypothesized that students from Congolese universities would be more exposed to the risk of sexual transmission of HIV than those from Japanese academic institutions in Kochi prefecture.

\section{Subjects and methods}

Participants

A multiple-center cross-sectional study was conducted among first- to third-year undergraduate university students from Japan (Kochi University Medical School, Kochi University School of Agriculture, Kochi University School of Human Sciences and Economics, Kochi National Nursing College) and the Democratic Republic of Congo (University Bel Campus and Songhwa University in Kinshasa; Institut Superieur de Commerce in Bas-Congo province, University of Kisangani in north-eastern Oriental province). In total, 1,747 students took part in the survey. Participants were enrolled simultaneously in Japan and the Democratic Republic of Congo from October 25 to November 27, 2009. Of the 1,747 students who eventually participated, there were 1,326 respondents, including 752 Japanese (297 medical and 455 non-medical students) and 574 Congolese students (193 medical and 381 non-medical students). The overall participation rate was $76 \%$ (57\% for the Japanese and $83 \%$ for the Congolese) and the ratio between medical and non-medical students was 3:5.

Questionnaire

The survey consisted of a structured self-administered anonymous questionnaire that had fifteen questions related to age, gender, marital status, sexual behavior, knowledge of different ways of sexual transmission of HIV, the use of condoms, and HIV serological testing. The survey was translated from English into Japanese (for Japanese participants) and into French (for Congolese participants). Participants' informed consent was obtained on the basis of a procedure that was officially approved by the authors' institutions. Surveyors distributed survey sheets to all students in each of the randomly selected classrooms from each campus, and students were advised that participation was voluntary. Those who did not consent to participate returned the questionnaire unanswered.

Data analysis

Statistical analyses were performed with the use of Stata software package, version 8 (StataCorp, TX, USA). The $\chi^{2}$ test was used to assess differences between Japanese and Congolese student groups, and between medical and non-medical student groups. A logistic regression analysis was performed, with the use of Stata software version 10 (StataCorp), to assess the association between variables and the correlation between potential predictors and HIV testing. $p$ values less than 0.05 were considered statistically significant.

\section{Results}

Characteristics of respondents

In total, there were 1326 respondents (752 Japanese, 574 Congolese), including 695 females $(52.4 \%)$ and 631 males (47.6\%) (Table 1).

There were 490 medical students (60.6\% Japanese, 39.4\% Congolese) and 836 non-medical students (54.4\% Japanese, $45.6 \%$ Congolese). Most respondents were single, 1071 students $(80.8 \%)$, whereas 255 were married (19.2\%). The mean age was $20.7 \pm 5.9$ years for the Japanese respondents and $21.9 \pm 3.9$ years for their Congolese counterparts.

Knowledge of STI as a risk factor for HIV infection

We searched to know whether Congolese students had accurate knowledge about STI as a risk factor for HIV in comparison with their Japanese counterparts. The proportion of Congolese (42\%) that had inaccurate knowledge about 
Table 1 Characteristics of the respondents

\begin{tabular}{lrrrrr}
\hline Characteristics & \multicolumn{2}{l}{ Japanese } & & \multicolumn{2}{l}{ Congolese } \\
\cline { 2 - 3 } \cline { 5 - 6 } & $n 1$ & $\%$ & & $n 2$ & $\%$ \\
\hline Age (years) & & & & \\
17-21 & 596 & 79.2 & & 345 & 60.1 \\
22 or more & 156 & 20.8 & & 229 & 39.9 \\
Total & 752 & 100.0 & & 574 & 100.0 \\
Gender & & & & \\
Female & 422 & 56.1 & & 273 & 47.66 \\
Male & 330 & 43.9 & 301 & 52.4 \\
Total & 752 & 100.0 & 574 & 100.0 \\
Medical & 297 & 39.5 & 193 & 33.6 \\
Non-medical & 455 & 60.5 & 381 & 66.4 \\
Total & 752 & 100.0 & 574 & 100.0 \\
Marital status & & & & \\
Single & 727 & 96.7 & 344 & 60.0 \\
Married & 25 & 3.3 & 230 & 40.0 \\
Total & 752 & 100.0 & 574 & 100.0 \\
\hline
\end{tabular}

$n 1$ number of respondents from Congolese universities, $n 2$ number of respondents from Japanese universities

STI was higher than that of Japanese students (31\%); but not significantly so $(p=0.106)$ (data not shown).

\section{Condom use with an occasional sex partner}

We assessed the students' practice in regard to this preventive measure with an occasional sex partner. A higher proportion of Congolese respondents, $57 \%$ (310/542) (vs. $15.5 \%$ for the Japanese side), reported never or not always using condoms in such a case, and only $43 \%$ (232/542) of Congolese respondents always used condoms (vs. $84.5 \%$ for the Japanese side) $(p<0.001)$. Of the Congolese medical students, $54 \%$ (58/108) (vs. 19.7\% for the Japanese side) did not always use condoms (Fig. 1) in sexual relations with an occasional sex partner.

These results show that, for Congolese respondents, the proportion of medical students with accurate knowledge of the risk of HIV in occasional sex was lower when compared with their Japanese counterparts, 46\% (50/108) and 69\% (128/186), respectively ( $p<0.001)$. In both the Congolese and Japanese cohorts, the proportion of medical students who always use a condom in sex with a partner of unknown HIV serological status was higher in those with a medical background as compared with non-medical students (Fig. 1).

Students' knowledge of five high-risk behaviors for sexual transmission of HIV

Of the high-risk behaviors related to the sexual transmission of HIV, we assessed the students' knowledge of the
Condom use in case of occasional sex partner

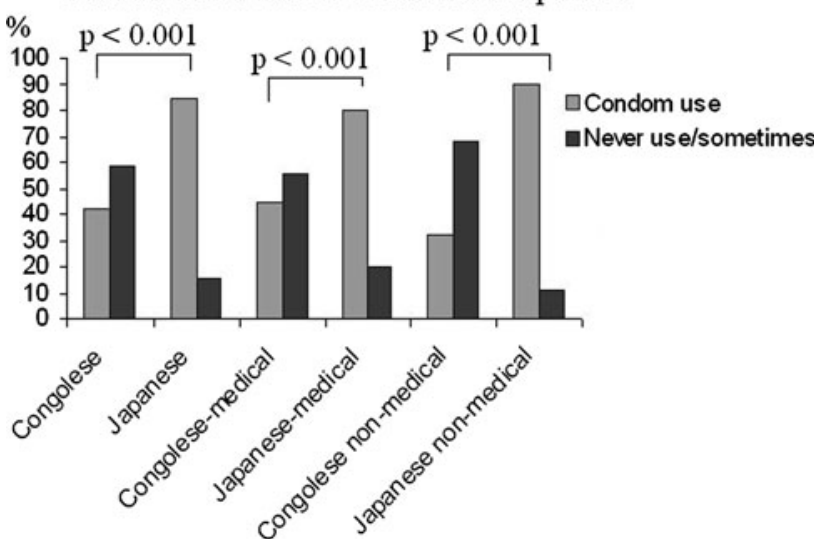

Fig. 1 Condom use with an occasional sex partner of unknown HIV status. $p, p$ value for $\chi^{2}$ test (Congolese vs. Japanese) The proportion of Congolese respondents who always use a condom in sex with an occasional partner was significantly lower, at $43 \%$, than that in their Japanese counterparts $(84.5 \% ; p<0.001)$. Among respondents from medical departments, 54\% (58/108) of Congolese never or do not always use condoms in such a case (vs. $19.7 \%$ for Japanese respondents; $p<0.001$ ), while the proportion of non-medical Congolese respondents (for sexually active individuals) who do not observe this requirement was higher, at $46 \%$, than that for the Japanese side $(11.4 \% ; p<0.001)$. Students were asked whether they always use condoms with an occasional sex partner

following: multiple sex partners (MSP), men sex with men (MSM), precocious sex, commercial sex work (CSW), and unprotected sex. Students had to answer whether each of these behaviors was a risk behavior for the sexual transmission of HIV. Regarding these high-risk behaviors, the overall knowledge level of Congolese respondents was significantly lower and more inaccurate than that of their Japanese counterparts (Fig. 2). Only 39.7\% of Congolese respondents (vs. $86.8 \%$ of Japanese respondents, $p<0.001$ ) had accurate knowledge about MSP; $9.8 \%$ (vs. $34.7 \%$ in Japanese respondents, $p<0.001$ ) had accurate knowledge about MSM; 9.9\% (vs. $13.3 \%$ in Japanese respondents, $p=0.036$ ) had accurate knowledge about precocious sex, and $34.4 \%$ (vs. $59.2 \%$ in Japanese respondents, $p<0.001$ ) had accurate knowledge about CSW. As for unprotected sex, which is well known as a high-risk behavior, $61.2 \%$ of Congolese respondents (vs. $74.1 \%$ for the Japanese side, $p<0.001)$ answered correctly.

Prevalence of HIV testing among the respondents

In this study, the proportion of sexually active Congolese respondents who would like their sex partner to undergo HIV testing was higher as compared with their Japanese counterparts, but not significantly so (89.8 vs. $82 \%$; $p>0.05$ ) (Fig. 3a). 


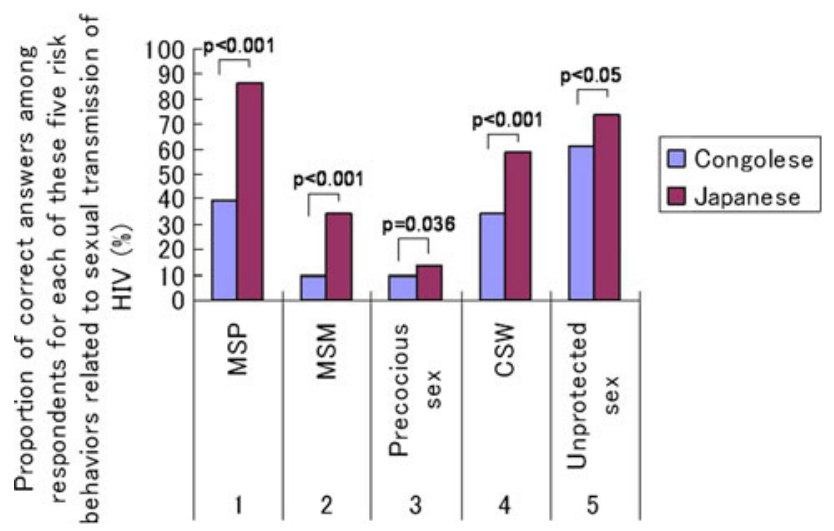

Fig. 2 Knowledge of five high-risk behaviors for sexual transmission of HIV. $p, p$ value for $\chi^{2}$ test (Congolese vs. Japanese). The figure shows the proportion of Congolese respondents who had accurate knowledge of five-high risk behaviors for the sexual transmission of HIV. Only $39.7 \%$ of the Congolese respondents (vs. $86.8 \%, p<0.001$ ) had accurate knowledge about MSP; 9.8\% (vs. 34.7\%; $p<0.01$ ) had accurate knowledge about MSM; 9.9\% (vs. 13.3\%; $p=0.036$ ) had accurate knowledge about precocious sex; and $34.4 \%$ (vs. $59.2 \%$; $p<0.001)$ had accurate knowledge about CSW. MSP multiple sex partners, $M S M$ men sex with men, $C S W$ commercial sex work

In addition, the prevalence of HIV testing among Congolese was higher than in the Japanese students, at 57.6 and $52.4 \%$, respectively, while the proportion of those who wanted to get tested (among the remaining students) was higher in Japanese respondents, at $45.2 \%$ (vs. $14.8 \%$ in Congolese students) (Fig. 3b). Taken together, the proportion of respondents who were either tested or willing to get tested for HIV was greater among Japanese respondents as compared to their Congolese counterparts (97.2 vs. $72.4 \%$, respectively; $p<0.01$ ).

Significant gender differences were observed for knowledge of risk factors and behaviors. An association was found between gender and knowledge about STI as a risk factor for HIV infection in Congolese respondents, with $59.1 \%$ of females having a better perception of the risk than males (54.7\%, $p=0.0009$ ) (vs. 72 and $61.2 \%$ in Japanese respondents, respectively; $p=0.0085$ ). Furthermore, Congolese female respondents tended to have accurate knowledge of the necessity to know about one's sexual partner's HIV serological status, as $92.7 \%$ of them ( $84.8 \%$ for males, $p=0.0109$ ) answered correctly. The same trend was noted in Japanese respondents, with $85.8 \%$ giving a correct answer to the question (vs. $73 \%$ of males), but the difference was not significant ( $p=0.053$ ) (Fig. 3). This shows that, in general, females have a better perception of the risk than males regarding HIV testing for their sex partners, as we expected.

Correlates of HIV testing among the respondents

In Congolese students, the lack of accurate knowledge of risk factors/behaviors such as unprotected sex $[39 \%$ of
A
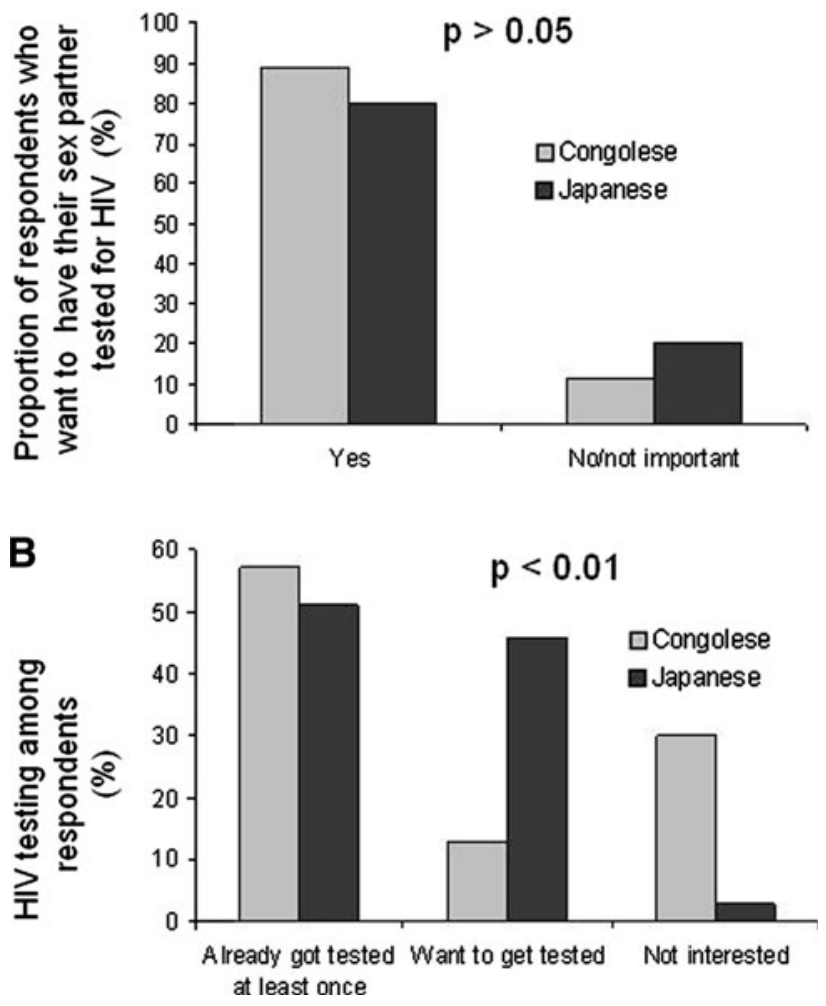

Fig. 3 Proportion of respondents who would like their sex partner to undergo HIV testing (a) and HIV testing status among the respondents (b). $p, p$ value for $\chi^{2}$ test (Congolese vs. Japanese). The figure shows that the proportion of sexually active Congolese respondents who would like their sex partner to undergo HIV testing was higher as compared with that in their Japanese counterparts, but not significantly so $(89.8$ vs. $82 \% ; p>0.05)$ (a) and the prevalence of HIV testing among Congolese was higher than that in the Japanese students, at 57.6 and $52.4 \%$, respectively, while the proportion of those who wanted to get tested (among the remaining students) was higher in the Japanese respondents, at $45.2 \%$ (b)

students having inaccurate knowledge and 58\% HIV tested; adjusted OR 1.3 (95\% CI 1.1-1.5); $p<0.05$ ], MSM [91.2\% of students with inaccurate knowledge and 56.7\% HIV tested; adjusted OR 1.2 (95\% CI 0.8-1.6); $p<0.05$ ], having 2 or more sex partners [60.1\% of students had inaccurate knowledge and $54.1 \%$ HIV tested; adjusted OR 2.8 (95\% CI 1.5-5.3); $p<0.01]$, oral sex [89.5\% of students had inaccurate knowledge and 57.3\% HIV tested; OR 1.2 (95\% CI 0.8-1.7)], and polygamy [56.7\% of students had inaccurate knowledge and 58.8\% HIV tested; adjusted OR 1.8 (95\% CI $0.9-3.4$ ); $p<0.05$ ] was associated with a higher prevalence of HIV testing. This suggests that most Congolese students with inaccurate knowledge of these risk factors/behaviors for sexual transmission of HIV had been tested for HIV at least once by the time of this survey. In contrast, the Japanese students tended to have accurate knowledge of most of these 
risk behaviors that were positively associated with HIV testing: unprotected sex [74\% students had accurate knowledge and 56.1\% HIV tested; adjusted OR 1.3 (95\% CI $1.1-1.5) ; p<0.05$ ], having 2 or more sex partners [86.8\% of students had accurate knowledge and 63.6\% HIV tested; adjusted OR 2.8 (95\% CI $1.4-5.3)$; $p<0.001]$, and polygamy [59.8\% had accurate knowledge and $62.5 \%$ HIV tested; adjusted OR 1.8 (95\% CI 0.9-3.4)] (Table 2). Regarding compulsory premarital HIV testing, few Japanese students, 33.8\%, had accurate knowledge; however, when compared with their Congolese counterparts, a higher proportion of these students $(63.5 \%)$ were HIV tested [adjusted OR 11.6 (95\% CI 1.3-100.4)] (Table 2).

In Congolese students, good knowledge of compulsory premarital HIV testing $[89.7 \%$ of students had accurate knowledge and 58.1\% HIV tested; OR 11.7 (95\% CI $1.4-100.5) ; p<0.01]$ and STI [58\% of students had accurate knowledge and $60.1 \%$ HIV tested; adjusted OR $1.2 ; 95 \%$ CI $1.1-1.5 ; p<0.05$ ] was positively associated with HIV testing. Taken together, these results suggest that Congolese respondents, in general, did not have accurate knowledge and good practice in regard to a number of major risk behaviors in their country or region, as compared with their Japanese counterparts, and tended to undergo HIV testing. This shows evidence of discrepancies between the Congolese and Japanese educated people in terms of perceptions of the risk of HIV infection, probably due to the influence of cultural factors.

Table 2 shows that, in Congolese students, there was a positive association between "HIV testing" and "having accurate knowledge of STI as a risk factor for HIV" (adjusted OR 1.2; 95\% CI 1.1-1.5; $p<0.05$ ); however, inverse associations were found between "HIV testing" and "accurate knowledge of MSM as a risk behavior for HIV" (adjusted OR 1.1; 95\% CI 0.8-1.6; $p<0.05$ ), "accurate knowledge of unprotected sex" (adjusted OR 1.3 ; 95\% CI 1.1-1.5; $p<0.05$ ), "inaccurate knowledge of polygamy as a risk behavior" (adjusted OR 1.8; 95\% CI $0.9-3.4 ; p<0.05$ ), and "having two or more sex partners as a risk behavior" (adjusted OR 2.8; 95\% CI $1.5-5.3 ; p<0.01)$. In Japanese students, a positive association was noted between "HIV testing" and "STI as a risk factor for HIV" (adjusted OR 1.2; 95\% CI $1.1-1.4 ; p<0.05)$, "unprotected sex as a risk behavior for HIV" (adjusted OR 1.3; 95\% CI 1.1-1.5; $p<0.05$ ), "having accurate knowledge of compulsory premarital HIV testing as a preventive measure for HIV" (adjusted OR 11.6; 95\% CI 1.3-100.4; $p<0.05$ ), and "accurate knowledge of having two or more sex partners being a risk behavior" (adjusted OR 2.8; 95\% CI 1.5-5.3; $p<0.01$ ).

\section{Discussion}

Though they have a high level of education, Congolese students are at higher risk of infection by HIV and other sexually transmitted diseases than their Japanese counterparts, because the majority of them do not have correct perceptions of the risks and accurate knowledge of local risk behaviors that may lead to the sexual transmission of HIV. For sexually active individuals, the use of condoms to prevent HIV infection has been promoted by health policy makers and organizations worldwide. The present study showed that $57 \%$ of Congolese students do not always use a condom when they have sex with an occasional partner, while only $15.5 \%$ of their Japanese counterparts do not observe this protective measure. And, regarding unprotected sex as a risk behavior for HIV, nearly $40 \%$ of the Congolese respondents ignored this fact, which is really risky as most of them are sexually active. In an awareness campaign conducted in Kinshasa, the capital city of the Democratic Republic of Congo, the National AIDS control program revealed the results of a survey that showed a high proportion $(86 \%)$ of young Congolese had had unprotected sex before the age of 20 in the year 2001 [7]. Similar trends have been reported in the United States among black Americans and Asian gays [8-10].

Precocious sex, unprotected sex, multiple sex partnership (MSP), and polygamy are among local risk behaviors for HIV in Sub-Saharan African countries, and HIV is mostly transmitted through sexual contact $[8,11]$. In the present study, the proportion of Congolese respondents who lack accurate knowledge of these risk behaviors $(80.1,39,60.1$, and $56.7 \%$, respectively) (Table 2) is alarming and this implies the need for change in their attitudes and perceptions of the risk of sexual transmission of HIV infection.

In most Sub-Saharan African countries, the above risk behaviors are reported to be among the main risk behaviors for HIV infection among young adults. Therefore, one would expect that the proportion of Congolese students with accurate knowledge would be higher than that in their Japanese colleagues. However, the Japanese students tended to have better knowledge of these risk behaviors. This reality shows the role of the cultural background in the spread of STI and HIV in a region where MSP and polygamy are considered as a cultural heritage.

The high proportion of Congolese respondents, $91.2 \%$ (vs. $65.3 \%$ in the Japanese group), who considered that MSM was not a risk behavior for sexual transmission of HIV could be explained by the fact that although this practice exists, it is not common in many regions of SubSaharan countries. Testing is important, as it may lead to an early diagnosis of HIV infection and early initiation of anti-retroviral treatment. In general, more than $50 \%$ of the Congolese and Japanese students (58.1 and 63.5\%, 


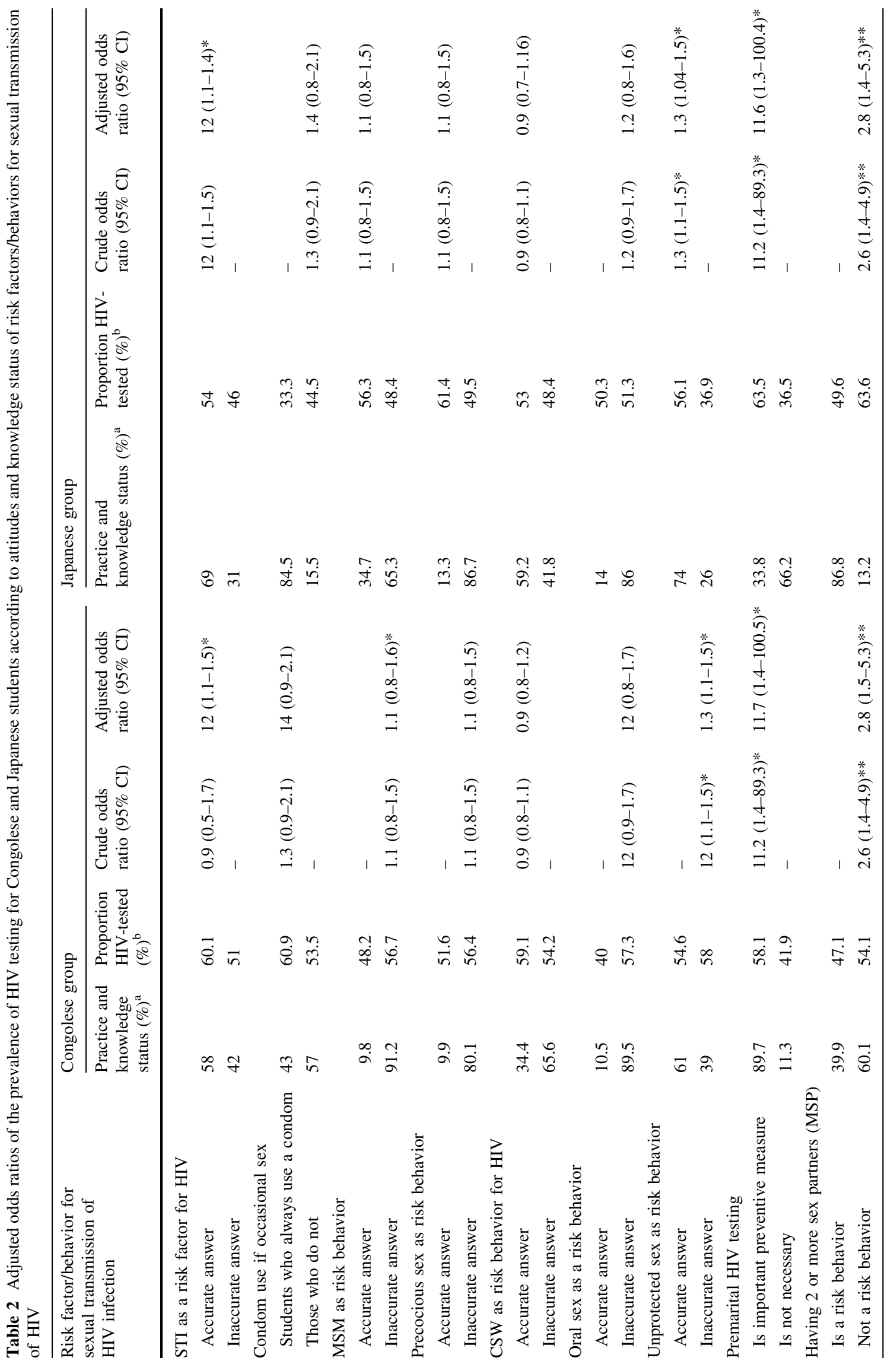


respectively) had been already tested for HIV, and more than $80 \%$ (89.8\% for Congolese vs. $82 \%$ for Japanese) of the respondents from each group said they would like their sex partner to undergo HIV testing. However, a gender discrepancy was observed, with female respondents having a relatively better perception of the risk $(59.3 \%$ of females vs. $53.9 \%$ of males for Congolese, $58.1 \%$ of females vs. $42.4 \%$ of males in Japanese). This fact is very encouraging because HIV prevalence in African countries is higher in women than in men, and women are known to be more vulnerable to HIV and other sexually transmitted diseases [12-16].

Premarital HIV testing should be promoted in awareness campaigns, as barriers to testing still exist. It has been reported that, of the 20,000 new HIV infections contracted annually in youth, $75 \%$ are in black and other minority groups; more than one third have not been tested for HIV and the majority is MSM youth and unaware of infection status [17]. The situation is quite similar to that in the Democratic Republic of Congo. The majority of Congolese students knew about the importance of premarital HIV testing; but, in contrast, only about half of them had been tested for HIV by the time the present study was conducted.

This study was limited by a cross-sectional design that limits causal inferences, and the sample size, though relatively big as compared to that in other similar studies, was quite restricted by the fact that in some other universities in the two provinces, Kinshasa and Bas-Congo (provinces that currently are safer in a country torn by what seems to be an interminable civil war), which could have participated in the survey, there were prolonged vacations, for different reasons. Also, on the other hand, students from Kochi prefecture do not necessarily represent all Japanese students. However, the sample of Japanese students surveyed in this study appears to be representative of first- to third-year students in Kochi city, a town in Kochi prefecture on the island of Shikoku whose HIV prevalence $(0.02 \%)$ is similar to the national prevalence [18]. In addition, Japanese students participating in this survey were from the main campuses (universities and colleges) and survey questionnaires were given to all students present in each of the randomly selected classrooms on the scheduled day for the survey. The same was true for Congolese participants (i.e., survey questionnaires were given to all students present in each of the randomly selected classrooms on the scheduled day for the survey). A future survey that includes students from different regions of Japan is essential.

Despite the above-mentioned limitations, this study showed significant differences in terms of accuracy of the perceptions of the risk of sexual transmission of HIV infection between two samples of educated populations, representing at least their respective places of residence. Congolese tended to have a lack of accurate knowledge of 
and lack of good practice regarding the main risk factors and behaviors that expose people to the sexual transmission of HIV as compared with their counterparts from the Japanese prefecture of Kochi.

To our knowledge, this study is the first comparative one to be conducted concurrently in high- and low-income countries and which demonstrates the possible role of socio-cultural factors, apart from illiteracy, related to HIV status in Sub-Saharan Africa, in particular, in the Democratic Republic of Congo. Awareness campaigns addressing local HIV-related risk behaviors should be reinforced in order to increase accurate personal risk assessments that may improve the perception of the risk of infection. HIV testing should be made accessible to a great number of sexually active populations. This implies the involvement of governments, health policy makers, and international organizations.

Acknowledgments The authors thank Assistant Professor Darren Lingley from the Kochi University School of Human Sciences and Economics; and Miss Rachel Tona Bilongi, Mr Samuel Vumi Kiaku, and Mr Jules Mbala, from the NGO Ligue pour la Promotion de la Sante (LIPS), for their support during the implementation of this study. This study was exclusively sponsored by the Department of Environmental Medicine, Kochi University Medical School, Japan.

\section{References}

1. World Health Organization (WHO). Status of global HIV epidemic. http://whqlibdoc.who.int/unaids/2008/9789291737116_ eng_Chapter2A.pdf (2008). Accessed Sep 2010.

2. Piot P, Bartos M, Larson H, Zewdie D, Mane P. Coming to terms with complexity: a call to action for HIV prevention. Lancet. 2008;372:845-59.

3. Maleta K, Bowie C. Selecting HIV infection prevention interventions in the mature HIV epidemic in Malawi using the mode of transmission model. BMC Health Serv Res. 2010;10:243-8.

4. Lazarotto A, Reichert MT, Venker C, Kramer AS, Sprinz E. HIV/ AIDS and middle aged: knowledge assessment of people from Vale do Sinos, Rio Grande do Sul State, Brazil. Cien Saude Colet. 2010;15:1185-90.
5. Bankole A, Singh S. Condom use and need for protection against HIV/AIDS. Current issues in comparative education. http://www. tc.columbia.edu/cice/articles/jved131.htm (2000). Accessed 10 Sep 2010).

6. Bankole A, Singh S, Woog V, Wulf D. Risk and protection, youth and HIV/AIDS in sub-Saharan Africa. http://www.guttmacher. org/pubs/riskandprotection.pdf (2001). Accessed on 10 Sep 2010.

7. Panafrican News Agency. 86 Percent of young Congolese had unprotected sex. http://allafrica.com/stories/200102280377.html (2001). Accessed 28 Feb 2001.

8. Quigley M, Munguti K, Grosskurth H, Todd J, Mosha F, Senkoro K, Newell J, Mayaud P, Ka-Gina G, Klokke A, Mabey D, Gavyole A, Hayes R. Sexual behaviour patterns and other risk factors for HIV infection in rural Tanzania: a case-control study. AIDS. 1997;11(2):237-48.

9. Futterman DC. HIV in adolescents and young adults: half of all new infections in the United States. Top HIV Med. 2005;13:101-5.

10. Choi KH, Han CS, Hudes ES, Kegeles S. Unprotected sex and associated risk factors among young Asian and Pacific Islander men who have sex with men. AIDS Educ Prev. 2002;14(6):472-81.

11. Kalichman SC, Cain D, Simbayi LC. Multiple recent sexual partnerships and alcohol use among sexually transmitted infection clinic patients, Cape Town, South Africa. Sex Transm Dis. 2011;38(1):18-23.

12. Laga M, Matendo R, Buve A. The situation in the cradle of AIDS: Congo and Central Africa. In: Celentano D, Bayrer C, editors. Public health aspects of HIV/AIDS in low and middle income countries. New York: Springer; 2008. p. 196.

13. UNAIDS. AIDS epidemic update. http://www.unaids.org/en/ knowledgecentre/resources/publications (2009). Accessed 14 Dec 2010.

14. Glynn JR, Caraël M, Auvert B, Kahindo M, Chege J, Musonda R, Kaona F, Buvé A, the study Group on the Heterogeneity of HIV Epidemics in African Cities. Why do young women have a much higher prevalence of HIV than young men? A study in Kisumu, Kenya and Ndola, Zambia. AIDS 2001;15:S51-60.

15. Masanjala W. The poverty-HIV/AIDS nexus in Africa: a livelihood approach. Soc Sci Med. 2007;64(5):1032-41.

16. MacPhail CC. Relative risk of HIV infection among men and women in a South African township. Int J STD AIDS. 2002;13(5):331-42.

17. Hightow-Weidman LB. Missteps, misunderstandings, and missed opportunities: HIV testing among young African Americans. Sex Transm Dis. 2009;36(9):584-91.

18. Nakaya T, Nakase K, Osaka K. Spatio-temporal modeling of the HIV epidemic in Japan based on the national HIV/AIDS surveillance. J Geogr Syst. 2005;7:313-36. 